\title{
Aplastische Anämien
}

\section{Eine und nur eine Antwort ist jeweils richtig!}

Welche Aussage zu aplastischen Anämien (AA) trifft zu?

Welches initiale Symptom bzw. welcher Laborbefund findet sich häufig bei idiopathischer $A A$ ?

Welcher Befund ist nicht mit einer schweren aplastischen Anämie (SAA) vereinbar?

Zur Diagnosesicherung
bzw. Differentialdiagnostik
einer $A$ A ist nicht dringend
erforderlich ...

Welche dieser Kombinationen ist die derzeitige immunsuppressive Standardtherapie bei $A A$ ?
A In Europa werden AA am häufigsten durch Einnahme von Thyreostatika verursacht.

$B$ In der Mehrzahl der Fälle basieren AA auf genetischen Defekten.

C Idiopathische AA treten gehäuft im 6 . Lebensjahrzehnt nach vorangegangenen zytostatischen Therapien auf.

D Bei AA handelt es sich im Gegensatz zu medikamentenassoziierten Agranulozytosen um häufige Erkrankungen.

E Idiopathischen AA liegt am ehesten eine aberrante Immunantwort mit T-lymphozytären Reaktionen gegen hämatopoetische Progenitorzellen zugrunde.

A Leukopenie mit relativer Lymphozytose

B Splenomegalie

C Eosinophilie und Basophilie im peripheren Blutbild

D Oropharyngeale Ulzerationen

E Multiple chromosomale Aberrationen der hämatopoetischen Knochenmarkzellen

A Retikulozyten $<20 \times 10^{9} / 1$

B Erhöhte Serumkonzentrationen von Bilirubin und LDH

C Petechien

D Neutrophile Granulozyten $<0,5 \times 10^{9} / 1$

E Hypozellularität des hämatopoetischen Knochenmarks (15\% der Norm)

A eine Knochenmarkbiopsie mit histopathologischer Begutachtung.

B eine Durchflusszytometrie auf GIP-Ankerproteine.

C eine zytogenetische Untersuchung von hämatopoetischen Knochenmarkzellen.

D eine in vitro-Kultur hämatopoetischer Stammzellen („colony forming unit assay“).

E ein mikroskopisches Differentialblutbild einschließlich Retikulozytenzahlbestimmung.

A allergische Reaktion

B Serumkrankheit mit synovialen Immunkomplexablagerungen

C Alopezie

D Lymphopenie mit dem Risiko opportunistischer Pneumocystis carinii-Infektionen

E Thrombophlebitis bei peripherer i.v.-Applikation

A Antilymphozytenglobulin, Ciclosporin A und Methylprednisolon

B Antilymphozytenglobulin, Oxymetholon und Ciclosporin A

C Antilymphozytenglobulin, Ciclosporin A, Methylprednisolon und Interleukin 3

D Antilymphozytenglobulin, Cyclophosphamid und Methylprednisolon

E Antilymphozytenglobulin und Methylprednisolon 
Welche Aussage zur Prognose von bzw. Spätfolgen nach AA trifft zu?

Infektionserkrankungen sind eine häufige Komplikation der AA. Welche Aussage zu Diagnostik, Therapie und Prophylaxe ist falsch?

Welche der nachfolgenden Aussagen trifft nicht zu?

Welche Aussage zur Thrombopenie bzw. Blutungskomplikationen bei AA trifft zu?

Für eine primäre allogene Knochenmarktransplantation bei AA muss/müssen ...
A Ein MDS mit zytogenetischem Nachweis einer Monosomie 7 nach AA ist im Vergleich zu einem MDS mit Trisomie 8 prognostisch deutlich günstiger.

B Die transfusionspflichtige Anämie mit konsekutiver Hämosiderose ist im Vergleich zu den Infekt- und den Blutungskomplikationen von entscheidender prognostischer Relevanz.

C Annähernd 40\% der Patienten entwickeln im Verlauf ihrer Erkrankung eine Paroxysmale Nächtliche Hämoglobinurie (PNH) mit deutlichen Hämolysezeichen.

D Die Rezidivrate erworbener AA nach erfolgreicher immunsuppressiver Standardtherapie beträgt ungefähr 30-40\% nach 10 Jahren.

E Das Risiko eines soliden Tumors nach immunsupressiver Therapie ist vernachlässigbar gering.

A Zur Prophylaxe einer Pneumocystis carinii-Infektion sollten VSAA-Patienten nach Therapie mit ALG einmal monatlich Pentamidin inhalieren.

B Bei neutropenischem Fieber unklarer Genese sollte eine ambulante Antibiotikatherapie mit einem oralen Cephalosporin der ersten Generation durchgeführt werden.

C Eine ausgeprägte Gingivostomatitis herpetica infolge einer reaktivierten HSV-Infektion sollte mit systemisch appliziertem Aciclovir behandelt werden.

D Eine generelle antibiotische Dauerprophylaxe ist bei Neutropenie nicht zu empfehlen.

E Bei therapierefraktärem Fieber in der Neutropenie mit Nachweis von pulmonalen Infiltraten ist eine intravenöse antimykotische Therapie (z.B. mit Amphotericin B) indiziert.

A Schwerwiegende Komplikationen einer HLA-identischen Geschwister-KMT bei AA sind Transplantatabstoßungen und Graft-versus-Host-Erkrankungen.

$B$ Ein MDS auf dem Boden einer erfolgreich behandelten AA entwickelt sich häufiger nach einer immunsuppressiven Therapie als nach einer Allo-KMT.

C Eine alternative Allo-KMT von einem unverwandten HLA-identischen Fremdspender ist bei jungen VSAA-Patienten nach Versagen der immunsuppressiven Therapie zu erwägen.

D Die langfristige Überlebensrate nach HLA-identischen Fremdspender-KMT bei SAA beträgt $80-90 \%$.

E Eine sekundäre Hämosiderose infolge langfristiger Erythrozytentransfusionen wirkt sich wahrscheinlich nachteilig auf die Prognose nach Allo-KMT aus.

A Das Risiko einer Alloimmunisierung durch Thrombozytentransfusionen ist relativ gering; auf eine Bestrahlung und Leukozytenfilterung der Blutprodukte wird verzichtet.

B Blutungskomplikationen sind selten und haben kaum prognostische Bedeutung.

C Bei hämorrhagischen Komplikationen sollten vorzugsweise gepoolte Thrombozytenpräparate und plättchenreiches Plasma transfundiert werden.

D Zur Blutungsprophylaxe werden Thrombozytentransfusionen ab Thrombozytenzahlen von $20 \times 10^{9} / 1$ generell empfohlen.

E Bei Fieber oder klinischen Blutungszeichen sollten die Thrombozytenzahlen einen Wert von $10-20 \times 10^{9} / 1$ nicht unterschreiten.

A der Patient jünger als 30 Jahre sein.

$B$ ein dizygoter Zwilling vorhanden sein.

C ein HLA-identischer Familien- bzw. HLA-identischer Geschwisterspender vorhanden sein.

D Transfusionen ausschließlich mit Zellpräparaten von Familienangehörigen erfolgen.

E ABO-Blutgruppen- und Rhesus-Identität von Donor und Empfänger vorliegen.

\section{Teilnahmebedingungen}

Für diese Fortbildungseinheit erhalten Sie drei Fortbildungspunkte im Rahmen des freiwilligen Fortbildungszertifikates. Hierfür

- müssen 9 der 11 Quiz-Fragen richtig beantwortet sein (bestanden hat auch, wer nicht weniger Fragen richtig beantwortet hat als der Durchschnitt aller Teilnehmer).

- müssen Seite 1 und Seite 2 des Evaluationsbogens vollständig ausgefüllt sein. Unvollständig ausgefültte Bögen können nicht berücksichtigt werden!

- muss eine CME-Wertmarke im Feld D (Evaluationsbogen) aufgeklebt oder Ihre DMW-Abonnement-Nummer eingetragen sein.

\section{Datenschutz}

Ihre Daten werden ausschließlich für die Bearbeitung dieser Fortbildungseinheit verwendet. Es erfolgt keine Speicherung der Ergebnisse über die für die Bearbeitung der Fortbildungseinheit notwendige Zeit hinaus. Die Daten werden nach Versand der Testate anonymisiert. Namens- und Adressangaben dienen nur dem Versand der Testate. Die Angaben zur Person dienen nur statistischen Zwecken und werden von den Adressangaben anonymisiert verarbeitet.

\section{Einsendeschluss ist der 5.11.2003}

(Datum des Poststempels). Die Zertifikate werden ab dem 12.11.2003 auf dem Postweg versandt. Von telefonischen Anfragen bitten wir abzusehen. Die richtige Auflösung des Quiz wird in der DMW Nr. 46/2003 (erscheint am 14.11.2003) veröffentlicht. 